\title{
CORRELATION VISUALIZATION FOR STRUCTURAL UNCERTAINTY ANALYSIS
}

\author{
Tobias Pfaffelmoser* \& Rüdiger Westermann
}

\author{
Technische Universität München, Computer Graphics and Visualization Group, Informatik 15, \\ Boltzmannstrasse 3, 85748 Garching, Germany
}

Original Manuscript Submitted: 09/01/2011; Final Draft Received: 07/24/2012

\begin{abstract}
In uncertain scalar fields, where the values at every point can be assumed as realizations of a random variable, standard deviations indicate the strength of possible variations of these values from their mean values, independently of the values at any other point in the domain. To infer the possible variations at different points relative to each other, and thus to predict the possible structural occurrences, i.e., the structural variability, of particular features in the data, the correlation between the values at these points has to be considered. The purpose of this paper is to shed light on the use of correlation as an indicator for the structural variability of isosurfaces in uncertain three-dimensional scalar fields. In a number of examples, we first demonstrate some general conclusions one can draw from the correlations in uncertain data regarding its structural variability. We will further explain, why an adequate correlation visualization is crucial for a comprehensive uncertainty analysis. Then, our focus is on the visualization of local and usually anisotropic correlation structures in the vicinity of uncertain isosurfaces. Therefore, we propose a model that can represent anisotropic correlation structures on isosurfaces and allows visual distinguishing of the local correlations between points on the surface and along the surface's normal directions. A glyph-based approach is used to simultaneously visualize these dependencies. The practical relevance of our work is demonstrated in artificial and real-world examples using standard random distributions and ensemble simulations.
\end{abstract}

KEY WORDS: uncertainty visualization, correlation, structural uncertainty, glyphs

\section{INTRODUCTION}

It is quite common to model the uncertainty at discrete points $\mathbf{x}_{i}$ in an uncertain scalar field by a multivariate random variable $\mathbf{Y}$, i.e., a vector consisting of multiple scalar random variables $Y\left(\mathbf{x}_{i}\right)$. The uncertainty at a point $\mathbf{x}_{i}$ is then given by a set of realizations of the random variable $Y\left(\mathbf{x}_{i}\right)$. The standard deviation of one of the random variables indicates the strength of the deviation of possible realizations from their respective mean value, independently of the means and deviations of random variables at any other point in the domain. Consequently, the standard deviation describes the local uncertainty but does not allow inferences on possible variations at different positions relative to each other.

For instance, let us assume that the means and standard deviations at two adjacent points are identical, but the realizations of the random variables at both points are stochastically independent. In this case it cannot be predicted whether there is a positive, zero, or negative derivative of the data between the two points, and consequently the values at these points relative to each other cannot be resolved. We will call this kind of uncertainty a structural uncertainty, since it is associated with the occurrence of particular structures in the data which are affected by the degree of dependency between the values at two or more data points. In particular, structural uncertainty covers all variabilities of geometric features (e.g. orientation, curvature, etc.) of multidimensional structures (e.g., surfaces). To avoid confusion, we note that in other communities the term "structural uncertainty" is used to indicate the uncertainty in the structure of a model.

\footnotetext{
${ }^{*}$ Correspond to Tobias Pfaffelmoser, E-mail: pfaffelmoser@tum.de, URL: http://wwwcg.in.tum.de/
} 
In the analysis of structural uncertainty it is thus necessary to first predict the statistical dependency between the random variables used to model the uncertainty, and then to analyze the possible structural variations of features in this field taking into account these dependencies. To achieve this, we assume the random variables used to model the uncertainty exhibiting a multivariate Gaussian distribution so that mean values and standard deviations exist, and the linear statistical dependency is given by the correlation. Nonlinear dependencies between random variables are not present for multivariate Gaussian distributions, and thus they are not covered by the methods proposed in this work.

For Gaussian-distributed random variables $X$ and $Y$ the mutual stochastic dependency is given by their correlation $\rho(X, Y)$, which ranges from -1 to 1 and characterizes the linear relation between the two variables. It is computed as $\operatorname{cov}(X, Y) / \sqrt{\operatorname{var}(X) \operatorname{var}(Y)}$, where $\operatorname{var}(X)$ and $\operatorname{var}(Y)$, respectively, denote the variance of $X$ and $Y$, and $\operatorname{cov}(X, Y)$ is the covariance between $X$ and $Y$. For a thorough discussion of the concepts of stochastic dependence and correlation, as well as approaches to compute correlation values from given realization sets, let us refer to [1-3].

A high positive correlation between two random variables indicates that realizations of both variables are likely to deviate into the same direction from their mean values. A strong negative (or inverse) correlation indicates that a strong positive deviation for one realization is likely to result in a strong negative deviation of the other realization, and vice versa. As the realizations of random variables in regions exhibiting very low correlation can be assumed independent of each other, the effect of uncertainty on a mean structure in such a region is to a large extent arbitrary. As a consequence, in such regions a high structural variation is caused by high uncertainty, and the resulting mean structures are not reliable. Contrarily, regions exhibiting high correlations are affected by "smooth" structural uncertainty, meaning that the uncertainty causes low frequent variations to occur more likely. Thus, high frequencies in the mean structures are not affected strongly and one can argue that they are, therefore, stochastically more stable. Correlation is thus a very important means to analyze the structural variability of particular features in uncertain data fields, and this property of correlation forms the basis of our investigations in this work.

Our first goal is to demonstrate the use of correlation as an indicator for structural uncertainty. For this purpose we have designed a number of examples, ranging from rather simple to quite complicated, which clearly show the interdependencies between (anisotropic) local correlation structures and the variability of specific features in the data. We will use these examples to strengthen the awareness of the relevance of correlation analysis for estimating possible structural changes of relevant features due to uncertainty.

Our second goal is to investigate methods for visualizing the structural variability of isosurfaces in uncertain threedimensional (3D) scalar fields. To achieve this, we propose a novel method for visualizing the correlation structures in the vicinity of such surfaces based on an anisotropic correlation model. By visually distinguishing between the local correlations between points on the surface and along the surface's normal directions, we enable an improved understanding of the geometric and topological variability of uncertain isosurfaces. The proposed visualization techniques can be used in addition to techniques which directly visualize the standard deviation [4,5], and thus strive for a comprehensive visualization of the full Gaussian covariance information.

The relevance of our work is demonstrated in artificial and real-world examples using standard random distributions and ensemble simulations. To effectively show the influence of correlation on the structural variability of features in multidimensional data sets, we have generated a number of synthetic data sets using specific random distributions to model uncertainty. In addition, we have used our methods to visualize the structural uncertainty in an ensemble of 3D temperature fields in the exosphere which was simulated by the European Centre for Medium-Range Weather Forecasts [6]. As we will show in this work, our proposed technique for correlation visualization allows us to conclude on the stochastic stability of the mean isosurface in this field, revealing regions which are strongly and weakly affected by structural uncertainty.

The remainder of this paper is as follows: In the next section we review previous work that is related to ours. In Section 3 we will have a closer look at the interpretation of correlation and its effect on certain features in uncertain scalar data sets. In Section 4 we will shed light onto requirements and challenges we see in correlation visualization, and we will propose a specific correlation model as well as a visualization technique that addresses some of these requirements. Furthermore, we will show its effectiveness in some examples, discuss advantages and limitations, and give some ideas on future research directions. 


\section{RELATED WORK}

Today, the expression of errors surrounding a derived value via error bars, variance plots, confidence intervals, or histograms is the norm in tables and two-dimensional (2D) graphs. In some scientific fields, uncertainty is considered a key quantity and domain-specific approaches for modeling and computing uncertainties have been proposed. Some of the most prominent examples demonstrating a rigorous treatment and analysis of uncertainty can be found in the theory of physical measurement [7] and inverse problems [8]. In computational science, statistical techniques have been applied directly via Monte Carlo simulation or by incorporating stochastic operator expansions into partial differential equation (PDE) formulations [9].

In the visualization of 3D scalar fields, however, the indication of accuracy in the displayed results is far from standard and has been mainly restricted to particular domains such as geographical information systems [10], seismology [11], and astrophysics [12], to name just a few. For an overview and taxonomy of uncertainty visualization let us refer to $[4,13,14]$. A web library providing a list of references to the major publications in the field of uncertainty visualization is maintained at [15].

One approach for representing uncertainty in 3D scalar fields is to provide it as secondary data that are visualized directly in addition to the primary data. It is quite common to use the deviation from a given mean value as a measure of the local uncertainty, and to visualize this measure by color and opacity, and animations thereof, texture, glyphs, or additional surface structures [16-20]. Such an embedding, however, even though it can provide a good indication of the local strength of uncertainty, is difficult to use to infer how the position and structure of specific features in the data are affected by uncertainty.

An alternative approach is to visually encode the positional variability that is caused on relevant features due to uncertainty. The majority of approaches has focused on visualizing the positional variation of isosurfaces and material boundaries in 3D uncertain scalar fields. The proposed strategies range from the visualization of confidence surfaces [5, 21] and flowlines [22], to surface diffusion techniques [23] and surface animations [24]. The most recent approaches [25-27] suggest modeling the uncertainty stochastically and deriving probability distributions for particular events associated with isosurfaces, such as the crossing of these surfaces along the view rays.

Only a very few approaches have explicitly addressed the visualization of data correlations due to uncertainty. [28] proposed a tool for the visualization of correlation between two 3D scalar fields via color mapping and slicing. The use of glyphs for the visualization of local covariance information was demonstrated in [29]. However, in none of these approaches was a correlation model considered, and the used glyphs were not adapted to particular surface structures in 3D data. The approach in [30] suggests a numerical technique for visualizing covariance and cross-covariance fields of two-dimensional results of stochastic simulations. The most recent technique [31] presents a cluster visualization approach for global correlation structures.

\section{STRUCTURAL UNCERTAINTY}

In this section we give examples showing the interdependencies between correlation and the structural variability of features in multidimensional scalar fields. In particular, we will shed light onto the interpretation of (anisotropic) correlation structures and thus emphasize the importance of correlation analysis for the prediction of possible effects of uncertainty on features in the data. By means of these investigations we will also demonstrate the importance of a correlation analysis and its visualization for making reliable assumptions on the uncertainty of geometric properties of certain features, e.g., position, orientation, and curvature for surfaces.

Experiment 1. Figure 1 illustrates a first example using 2D curves that are affected by uncertainty to demonstrate how correlation information can be used for analyzing structural uncertainty. In this example, at every position $x_{i}$ in a set of equally spaced positions in the $x$ domain, multivariate normal distributed random variables $Y\left(x_{i}\right)$ with smoothly varying means but the same standard deviation were used to generate sets of $y$ values for every $x_{i}$. The green curve shows the mean $y$ values at every $x_{i}$. The confidence region for $\pm \sigma$ is enclosed by two blue dashed lines. The red curves always show lines for three possible realizations of the $y$ values at the points $x_{i}$ for very strong (a) and weak (b) local correlations between adjacent random variables $Y\left(x_{i}\right)$ and $Y\left(x_{i+1}\right)$. 

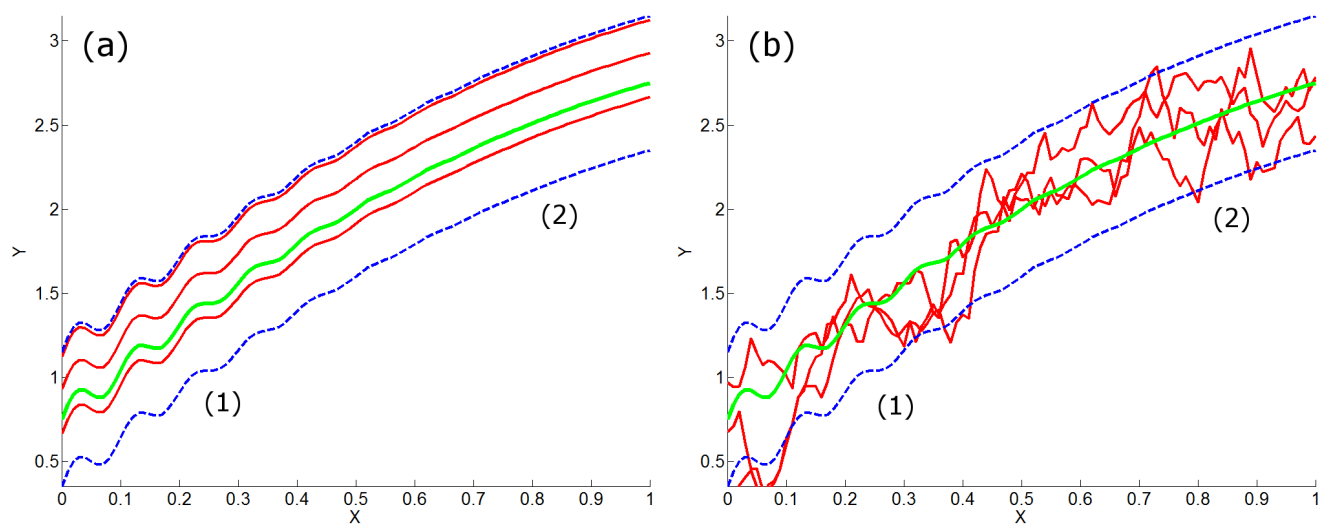

FIG. 1: Illustration of the structural variability of a 2D curve (green) given by a set of points $\left(x_{i}, y_{i}\right)$, where each $y_{i}$ represents the mean of the realizations of a multivariate normal distributed random variable at $x_{i}$. While the mean values vary for different $x_{i}$, the standard deviation remains constant. Blue dashed lines enclose confidence region around the mean curve. Three possible line realizations (red) are shown for very strong (a) and weak (b) local correlation between random variables at adjacent points.

In region (1), the expectation values of the normal distributions used to generate the $y$ values were chosen such that the mean curve shows higher frequencies than in region (2). Due to the strong correlation between the random values at adjacent positions, the three possible occurrences of curves in (a) show the same structural behavior as the mean curve in (a), while in (b) these structures disappear completely due to the low correlation. Strong correlation effectively keeps adjacent realizations of the random variables used to model the curves close together, while they can occur rather unconstrained with respect to each other in regions of low correlations. The knowledge of the mean and the standard deviation of the normal distributions alone does not allow inference on the structural behavior of the resulting realizations.

Experiment 2. Our next example demonstrates the use of correlation analysis for predicting the variability of the orientation of curves that are affected by uncertainty. In a first experiment, at two points positioned at $x=0$ and $x=1$ on the $x$ coordinate axis a bivariate normal distributed random variable was used to compute sets of $y$ values. The green line in Figs. 2(a)-2(c) shows the linear interpolation between the mean $y$ values at the two points. The linearly interpolated confidence region is enclosed by the blue dashed lines. The red curves always show the linear interpolation between two possible realizations of the data values at the two points. However, in Figs. 2(a)-2(c), respectively, random values were generated using a correlation of $\rho=-1, \rho=0$, and $\rho=1$ between these values.

To analyze the variability of the orientation of the line connecting the values at $x=0$ and $x=1$, in Figs. 2(d)-2(f) we show the probability density functions of the derivative of this line for the different correlations. The probability density function was computed by taking the derivatives for all pairs of realizations at the two points and counting their occurrence. In Fig. 2(g), the relation between the correlation and the variance of the distribution of the derivative is shown. The maximum possible variation in the derivative is observed for $\rho=-1$ (inverse correlation), the minimum for $\rho=1$.

From the experiment above, it becomes clear that the correlation has a strong impact on the variability of structural properties of certain features in the data, such as a feature's orientation. The reason is that correlation describes the behavior of random variables at different sample points relative to each other and thus constrains the amount of variation between the possible data values at these points.

Experiment 3. The aforementioned considerations can easily be transferred to higher dimensions to analyze the structural variability of isosurfaces in a 3D scalar field. To demonstrate this, we have pursued an experiment where at every vertex of a Cartesian 3D grid a set of random scalar values was generated using a multivariate normal distributed random variable with constant standard deviation and linearly increasing mean (starting at 0 ) along the $z$-grid dimension. The random values were generated with respect to a specific anisotropic correlation structure. The 

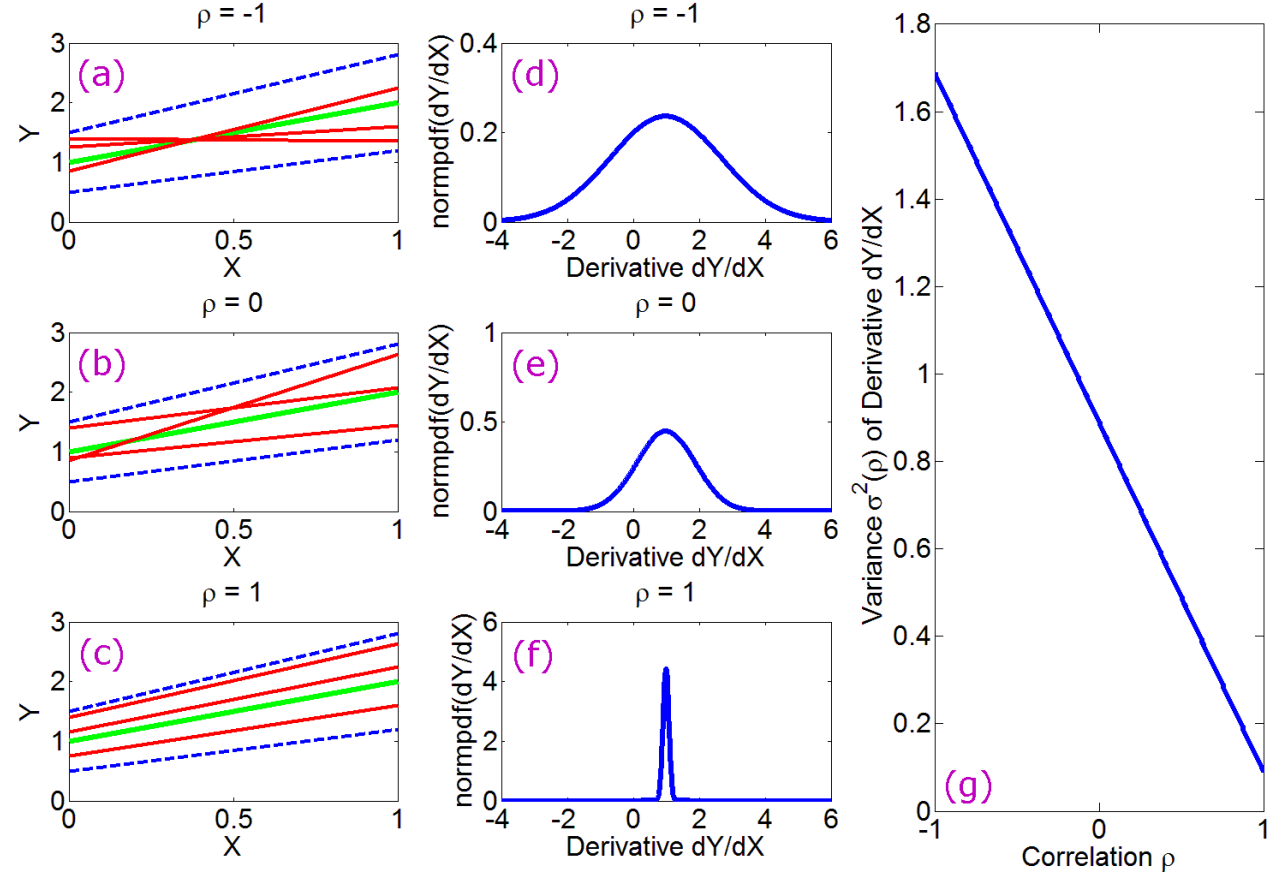

FIG. 2: In (a)-(c), for two bivariate normal distributed random variables $Y(0)$ and $Y(1)$ the means (connected by a green line) and the confidence region (enclosed by two blue dashed lines) are shown. Red lines show three realizations for correlations $-1,0$, and 1 , respectively. In (d)-(f) the Gaussian probability density for the derivative between $Y(0)$ and $Y(1)$ is illustrated. (g) illustrates the relation between correlation values and variance of the derivative distribution.

computed mean values were then stored at the grid vertices and an isosurface for a selected value was rendered [see Fig. 3(a)]. In Fig. 3(b), the confidence volume containing all points that belong to the surface with a certain probability is enclosed by two stochastic distance surfaces (transparent white). The blue line represents the contour of the mean surface, the colors encode the distance from the mean position in numbers of standard deviations $\sigma$-from $0 \sigma$ (green) to $\pm 1 \sigma$ (red). For details on this visualization mode the reader is referred to [25]. Notably, the visualization
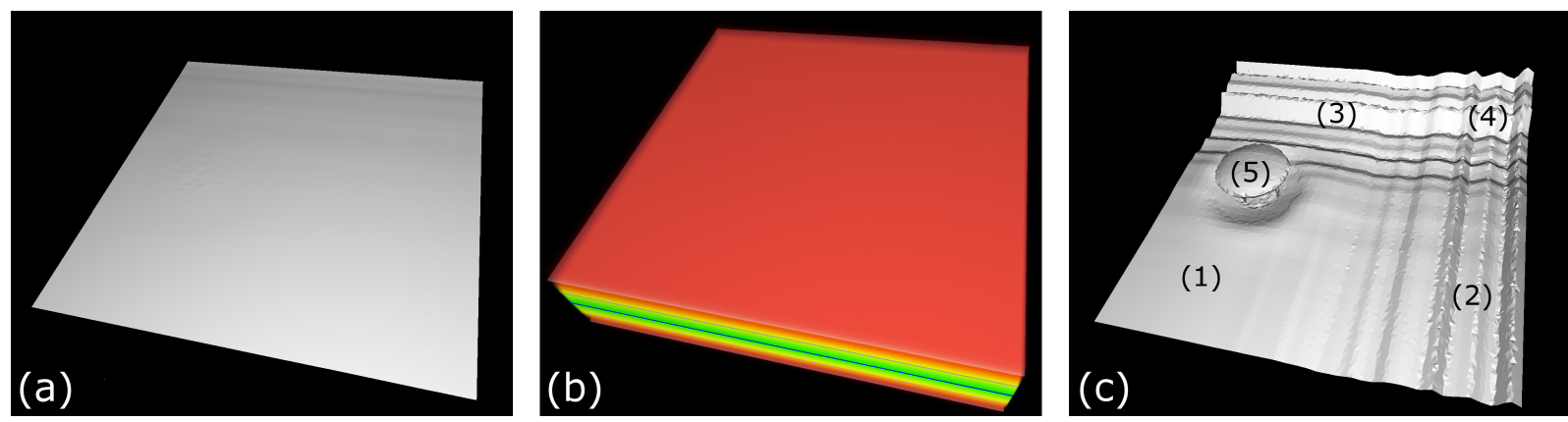

FIG. 3: Mean isosurface situated in the $x-y$-coordinate plane is shown in (a). For an assigned constant standard deviation the confidence region around the mean surface is visualized in (b). The structural variation, caused by the assigned correlation structure, is visible in the surface realization shown in (c). Strong homogeneous correlation was assigned to region (1). In (2) and (3) the correlation magnitude was lowered in the $x$ and $y$ direction, respectively. In region (4), low correlation in both tangential direction was applied. Around (5), low correlation along the surface normal direction was modeled. 
in Fig. 3(b) does not allow inference on the possible structural variations of the surface; it can only tell in which region of the domain the surface can be expected with a certain probability.

In Fig. 3(c), the occurrence of the surface in Fig. 3(a) for one possible realization of the random values at the grid points is shown. In region (1), the correlations between the random variables in the $x, y$, and $z$ direction were all set to 1. Consequently, the structure of the mean surface is not changed [cf. Fig. 1(a)]. In region (2), the correlation in the $x$ direction was lowered with respect to region (1). This results in oscillations of the surface in the $x$ direction. As the correlation in the $y$ direction is still 1 , the structure does not change along the $y$ direction and stiff ridges occur along this direction. The same correlation setting was applied to region (3), but this time with a lower value in the $y$ direction. In region (4), the correlations along $x$ and $y$ were lowered simultaneously, but the correlation in the $z$ direction was kept at 1 . This means that the surface can possibly oscillate, but since the values in the 3D scalar field are still highly constrained to each other along the $z$ direction, the surface remains connected. Moreover, the surface still indicates the exact location of the transition between scalar values lesser and greater than the selected isovalue.

The surface structure changed completely when the correlation in the $z$ direction was made significantly smaller than the correlation in the other two directions. This is shown in region (5). The lower correlation in the surface normal direction can result in several closely located but not necessarily connected surfaces, ${ }^{1}$ i.e., the transition surface becomes fuzzy and does not have the separating property it had before. The example shows that a visualization that is purely based on uncertainty parameters like standard deviations [cf. confidence volume in Fig. 3(b)] does not allow inference on the geometric structures, which is likely to occur in realizations of the underlying probability model.

Experiment 4. In our last experiment, we outline the importance of correlation analysis in the context of inverse problems, e.g., 2D travel-time tomography, where physical measurements are used to infer the media through which wave forms are traveling. In this experiment, we model a geophysical setup, where receivers close to the earth's surface measure the arrival of pressure waves originating from earthquake sources with known positions in the earth's interior. The goal is to determine parameters describing the travel velocity of the waves in certain parts of the interior, based on measured wave travel times between each source-receiver pair.

According to geophysical realities, the seismic travel-time inverse problem is nonlinear, as seismic waves do not follow straight paths in a heterogeneous medium and bend continuously as the velocity parameters of the medium vary. For simplification, we will abstract these real physical conditions and assume a linear inverse problem with straight pressure wave travel paths between source and receiver. This abstraction complies with the interpretation of the inversion as a first-order correction to a homogeneous medium. Thus, even though real nonlinear physical conditions are abstracted, the following considerations and interpretations can be adapted to every kind of inverse process step where a linearization takes place.

Mathematically, the abstract problem can be formulated by the system of equations

$$
\mathrm{Gm}=\mathbf{d},
$$

where $\mathbf{G}$ represents the problem-specific linear operator, $\mathbf{d}$ is the linearized vector of measured data (travel times), and $\mathbf{m}$ is the linearized vector of unknown parameters. The goal is to compute $\mathbf{m}$ for a given $\mathbf{d}$.

In travel-time tomography, the inverse problem is often underdetermined and does not have a unique solution. For linear inverse problems and by using Bayesian probability theory, however, the solution $\mathbf{m}$ can be described by a multivariate normal probability density function. This function is characterized by a mean $\overline{\mathbf{m}}$ and a covariance matrix $\mathbf{C}_{\mathbf{m}}$. For more details on the physical theory and model setup, refer to [32] and [8].

Figure 4(a) shows a rectangular domain discretized by a triangular grid [shown in Fig. 4(b)], which models a cross section of the earth. The components of the parameter vector $\mathbf{m}$ are placed at the grid vertices. The parameters define for each vertex a slowness value (inverse of velocity: time/distance). In the upper and lower part of the domain, respectively, five receivers (green diamonds) and seven sources (red stars) are placed. The travel paths are illustrated by black lines. The travel time for each path is obtained by integrating the slowness parameters along the path (linear interpolation in each triangle). As all parameters in $\mathbf{m}$ appear linear in the integrals (Simpson quadrature), the integrals

\footnotetext{
${ }^{1}$ The regular spherical surface structure results from the specific random number generation process we have used to assign anisotropic correlation structure.
} 

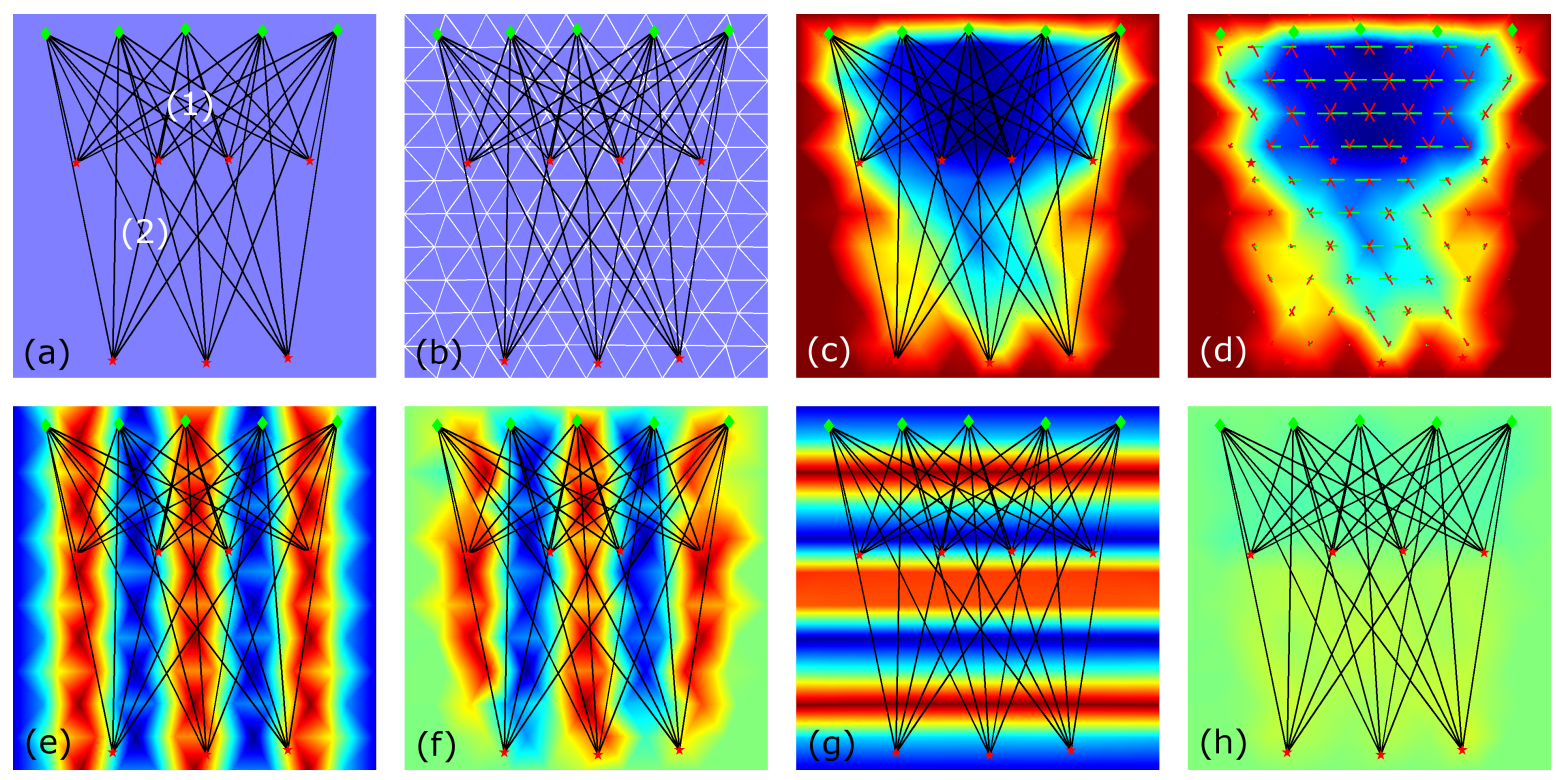

FIG. 4: Travel-time tomography experiment. (a), (b) Emitter (red stars)-receiver (green diamonds) pairs and domain triangulation. (c) Color mapping of normalized standard deviations to mean solution of inverse problem $([0 ; 1]$ to [blue $\rightarrow$ red]). (d) Visualization of correlation between adjacent grid points (line length corresponds to correlation strength, green/red indicate positive/negative correlation). (e), (g) Two different parameter configurations (color coded) used in a forward computation. (f), (h) Inverse computation of parameters from the results of forward computation.

can be expressed by a matrix $\mathbf{G}$ representing the operator in Eq. (1). The vector $\mathbf{d}$ contains the travel times for all paths.

Intuitively, one would expect that in regions which are covered by a large number of rays, e.g., in (1), there is a lower uncertainty in the computed values than in regions which are poorly sampled by rays, e.g., in (2), since less information is obtained there. To confirm this, we have first computed the covariance matrix $\mathbf{C}_{\mathbf{m}}$ from the given operator $\mathbf{G}$ (for details refer to [8]), and we have derived the standard deviations of the probabilistic solutions for the slowness values from this matrix, i.e., the square roots of entries on the main diagonal. In (c), these normalized standard deviations are color-coded via the mapping $[0,1] \rightarrow[$ blue $\rightarrow$ red $]$. The coloring clearly indicates that regions with higher ray density are affected by lower uncertainty (close to zero).

To demonstrate the importance of correlation for the analysis of the uncertainty in the computed results, we have performed the following experiment: First, we have specified two artificial slowness configurations $\mathbf{m}_{h}$ and $\mathbf{m}_{v}$ with periodic horizontal and vertical change of slowness values, respectively, and we have performed forward computations to obtain data vectors $\mathbf{d}_{h}$ and $\mathbf{d}_{v}$. In Figs. 4(e) and 4(g), these slowness parameters are color coded. Second, with $\mathbf{d}_{h}$ and $\mathbf{d}_{v}$ we have solved two inverse problems, resulting in two probabilistic solutions for the slowness values. Their means $\overline{\mathbf{m}}_{h}$ and $\overline{\mathbf{m}}_{v}$ are color coded in Figs. 4(f) and 4(h) using the same color mapping as in Figs. 4(e) and 4(g). As can be observed, only $\overline{\mathbf{m}}_{h}$ is close to its initial distribution, while $\overline{\mathbf{m}}_{v}$ shows almost no similarity or relevant structure, although the uncertainty in Fig. 4(c) in both solutions is the same.

In order to understand this phenomenon, we have to look at the correlations between the values at adjacent points, which are contained in the covariance matrix $\mathbf{C}_{\mathbf{m}}$. Therefore, in Fig. 4(d) we have visualized the pairwise correlations between the values at every grid point and its one-ring neighbors. Visualization is performed by drawing lines from the vertex to every adjacent vertex, where the lengths of these lines are linearly related to the magnitude of the respective correlation value. For $|\rho|=1$ the lines on either side of an edge meet at the edge's center. The lines are green for $\rho \geq 0$ and red for $\rho<0$. The visualization clearly indicates strong positive correlations in the horizontal direction and strong negative correlations in the vertical direction. 
As discussed above, for strong positive correlations, high frequent structures in the data are better resolved, as they are only affected by low frequency uncertainty. For inversely correlated regions, no reliable assumptions can be made. Thus, the example clearly shows that only structural changes in horizontal direction can be resolved by the inverse computation. A variation of parameters in vertical direction cannot by "seen" by this tomographic setup. So the correlation visualization in Fig. 4(d) already provides a clear indication that the data set is affected by a strong vertical but only a low horizontal structural uncertainty. This information is not revealed by visualizing only absolute uncertainty values [standard deviations in Fig. 4(c)]. As a consequence, it can be concluded that the consideration of correlation information and its visualization can significantly help to avoid misinterpretations that would occur otherwise.

\section{CORRELATION VISUALIZATION}

In the previous section, we have tried to demonstrate the relevance of correlation for uncertainty analysis. As a consequence, we believe that correlation visualization is a necessary ingredient in uncertainty visualization to facilitate a more reliable prediction of the possible effects of uncertainty on specific features in uncertain data. In this section, we will shed light onto the particular requirements we see in correlation visualization, and we will propose a correlation visualization technique that addresses some of these requirements.

\subsection{Requirements}

In real-world applications, correlation data are often given as correlation matrices which can be either directly computed (cf. Example 4 in Section 3) or estimated from an ensemble data set. If the data are given on a grid with $n$ grid points, a correlation matrix would have $n^{2}$ entries, of which $0.5 n(n-1)+n$ would be different. For large multi-dimensional grids, this $O\left(n^{2}\right)$ memory and computational complexity has to be reduced significantly before correlation visualization becomes feasible.

However, one difficulty in correlation analysis is that both local and global correlation effects have to be considered and the anisotropic nature of correlation makes it difficult in general to represent it with only a few values. By restricting the analysis to local effects, the first problem can be addressed. A possible solution for the second problem builds upon the development of a correlation model that can efficiently represent anisotropic structures, for instance, by trying to approximate these structures via a suitable basis transformation into a compact representation. Such a model can then be employed for the visualization of correlation, but additional requirements have to be considered. In particular, the direction dependent differences in correlation strength have to be depicted by an appropriate visual mapping. Such a mapping, besides being able to indicate the correlation ratios into different directions, should also allow a comparison of the absolute correlation strengths at different regions in the underlying domain.

Another challenge in correlation visualization arises from the general difficulty of finding a suitable visual mapping for complicated multidimensional structures. Even without considering uncertainty, the creation of approaches for mapping such structures onto 2D pixel images in an intuitive way is extremely difficult, and it is not clear by far how additional correlation structures can be integrated into conventional uncertainty visualization approaches. Therefore, as we have demonstrated in our third experiment in Section 3, one strategy is to restrict the correlation analysis to specific features in the data, such as isosurfaces in 3D scalar fields. In such a scenario, one can restrict the analysis to the local correlations along and in close vicinity to the feature, possibly augmented by contextual visualizations of the surrounding structures.

\subsection{Distance-Dependent Correlation}

We will now introduce a local anisotropic distance-dependent correlation model, which forms the basis of the introduced visualization approach. As we will show, this model has some specific advantages, and it allows transforming of correlations that are expressed in other models into this model straightforwardly. It considers the strength of positive local correlations for analyzing a given data set with respect to structural uncertainty as discussed in Section 3 . 
In the following, we assume a 3D Cartesian grid structure $\mathbb{C}_{p q r}=(\mathbf{x})_{i j k}$ with $1 \leq i \leq p, 1 \leq j \leq q, 1 \leq k \leq r$, which is attributed by a mean value and mutual correlation values for neighboring vertices at every grid vertex. These data can be linearly interpolated from values in any arbitrary grid structure. The grid spacing can be specified interactively by the user in order to achieve different glyph resolutions.

With this visualization approach we are aiming for an analysis of correlation effects in 3D scalar data sets. Usually, such data sets are assumed to be at least continuous and smooth up to a certain degree. This requires that the closer two spatial points in the underlying domain are, the higher the correlation should be between the random variables that are used to model the uncertainty at these points. To achieve this, the correlation is often modeled by a spatial distance-dependent correlation function [8]. One typically uses the exponential correlation function (ECF)

$$
\rho\left[Y\left(\mathbf{x}_{i j k}\right), Y\left(\mathbf{x}_{l m n}\right)\right]=\exp \left(-\tau\left\|\mathbf{x}_{i j k}-\mathbf{x}_{l m n}\right\|\right), \mathbf{x}_{i j k}, \mathbf{x}_{l m n} \in \mathbb{C}_{p q r},
$$

which assigns higher correlations to random variables of points with smaller Euclidean distance and lower correlations to points which are more distant from each other. If the correlation strength parameter $\tau$ is defined locally for each point in $\mathbb{C}_{p q r}$, the ECF becomes

$$
\rho\left[Y\left(\mathbf{x}_{i j k}\right), Y\left(\mathbf{x}_{l m n}\right)\right]=\exp \left\{-0.5\left[\tau\left(\mathbf{x}_{i j k}\right)+\tau\left(\mathbf{x}_{l m n}\right)\right]\left\|\mathbf{x}_{i j k}-\mathbf{x}_{l m n}\right\|\right\} .
$$

The ECF meets the intuitive assumption that scalar realizations behave more similarly if the respective sample points are closer in Euclidean space. We can consider this as a kind of local stochastic continuity.

The distance-dependent correlation model assumes isotropic correlations at every grid point. To model anisotropic correlations, $\tau$ can be made dependent on a specific direction. For a unit vector $\mathbf{r}$, the parameter $\tau$ at point $\mathbf{x}_{i j k}$ in direction $\mathbf{r}$ is then given by $\tau\left(\mathbf{x}_{i j k}, r\right)=\mathbf{r}^{\top} \mathbf{T}\left(\mathbf{x}_{i j k}\right) \mathbf{r}$, where $\mathbf{T}$ is the rank-2 parameter tensor that models the anisotropy. This tensor can either be derived from the correlations in the data samples, or it can be specified based on a priori knowledge. The adapted correlation model becomes

$$
\begin{aligned}
\rho\left(Y\left(\mathbf{x}_{i j k}\right), Y\left(\mathbf{x}_{l m n}\right)\right) & =\exp \left[-\frac{\left(\mathbf{x}_{i j k}-\mathbf{x}_{l m n}\right)^{\top} \overline{\mathbf{T}}\left(\mathbf{x}_{i j k}-\mathbf{x}_{l m n}\right)}{\left\|\mathbf{x}_{i j k}-\mathbf{x}_{l m n}\right\|}\right] \\
\overline{\mathbf{T}} & =0.5\left[\mathbf{T}\left(\mathbf{x}_{i j k}\right)+\mathbf{T}\left(\mathbf{x}_{l m n}\right)\right] .
\end{aligned}
$$

One advantageous use of the distance-dependent tensor model is to transform correlation data into this model and thus to avoid the explicit storage of a correlation matrix. Therefore, at every grid point $\mathbf{x}_{i j k}$ in $\mathbb{C}_{p q r}$ a tensor is computed from the correlations to its (at most) 26 neighbors

$$
\mathbb{N}\left(\mathbf{x}_{i j k}\right):=\left\{\mathbf{x}_{l m n} \in \mathbb{C}_{p q r} \mid \mathbf{x}_{l m n} \neq \mathbf{x}_{i j k}, \max (|i-l|,|j-m|,|k-m|)=1\right\} .
$$

If $\mathbf{x}_{i j k}$ is not a border point of the grid $\mathbb{C}_{p q r}$, then for every point $\mathbf{n}_{h} \in \mathbb{N}\left(\mathbf{x}_{i j k}\right)$ there exists an opposing point $\hat{\mathbf{n}}_{h} \in \mathbb{N}\left(\mathbf{x}_{i j k}\right)$ such that $\mathbf{x}_{i j k}=0.5\left(\mathbf{n}_{h}+\hat{\mathbf{n}}_{h}\right)$. At most 13 such pairs of $\mathbf{n}_{h}$ and $\hat{\mathbf{n}}_{h}$ with $h \in\{1,2, \ldots, 13\}$ can be built. By using the correlation model (2) and averaging the correlation against opposing neighbors one obtains 13 equations:

$$
\begin{aligned}
\mathbf{r}^{\top} \mathbf{T r} & =\frac{-\log \left(0.5\left\{\left|\rho\left[Y\left(\mathbf{x}_{i j k}\right), Y\left(\mathbf{n}_{h}\right)\right]\right|+\left|\rho\left[Y\left(\mathbf{x}_{i j k}\right), Y\left(\hat{\mathbf{n}}_{h}\right)\right]\right|\right\}\right)}{\left\|\mathbf{n}_{h}-\mathbf{x}_{i j k}\right\|} \\
\mathbf{r} & =\frac{\mathbf{n}_{h}-\hat{\mathbf{n}}_{h}}{\left\|\mathbf{n}_{h}-\hat{\mathbf{n}}_{h}\right\|}, \quad \mathbf{n}_{h} \in \mathbb{N}\left(\mathbf{x}_{i j k}\right), \quad h \in\{1,2, \ldots, 13\}
\end{aligned}
$$

If $\mathbf{x}_{i j k}$ is a border point, not for every $\mathbf{n}_{h} \in \mathbb{N}\left(\mathbf{x}_{i j k}\right)$ does there exist an opposing point. In this case, averaging is not performed in (6) and only the $\operatorname{logarithm} \log \left(\left|\rho\left[Y\left(\mathbf{x}_{i j k}\right), Y(\mathbf{n})\right]\right|\right)$ is used. Note that only the magnitudes of the correlations are used as the ECF only models the correlation strength. For integrating inverse correlation, another (global) model would be necessary, which was not considered in our work so far.

The entries of the $3 \times 3$ matrix $\mathbf{T}$ are denoted as $t_{i j}$. As $\mathbf{T}$ is symmetric and represents a rank- 2 tensor, only the six values $t_{11}, t_{22}, t_{33}, t_{12}, t_{13}, t_{23}$ have to be determined. These values appear linearly in the 13 (or less if $\mathbf{x}_{i j k}$ is a 
border point) equations (6). So one can build a $13 \times 6$ overdetermined linear system, which can be solved using a least squares approach and the normal equation [33].

The ECF model provides several advantages, amongst others also for visualization purposes. First, using a local approach reduces the memory requirement for storing correlation information to some extet: if the correlation grid has $m=\left|\mathbb{C}_{p q r}\right|=p q r$ entries, the respective correlation matrix has to store $m^{2}$ local and global correlation values. If only local values are stored, approximately 13 values per point in $\mathbb{C}_{p q r}$ would be needed for the 26-element neighborhood system described above. This amounts to a total of $13 \mathrm{~m}$ correlation values. The rank- 2 tensor model halves this amount to a total of $6 m$ values. This meets the proposed requirement from Section 4.1 for a memory consumptions of $O(m)$.

An additional reason for using the ECF model is its integration of Euclidean distances. To understand this, let us assume that instead of the local $\tau$ tensor, the local correlation values are used at every grid vertex. In this case, the local correlation in every direction depends on the resolution of the correlation grid $\mathbb{C}_{p q r}$, and if this grid has a high resolution, the correlation between neighboring points would approach 1 everywhere. Notably, the computation of $\mathbf{T}$ is independent of the grid resolution, as the Euclidean distances to the neighbors are taken into account for computing $\mathbf{T}$. Thus, correlation is set in relation to Euclidean distances. Furthermore, using the parameter tensor allows the computation of correlations for a user-specified distance (cf. Section 4.3). With a stored correlation tensor for a fixed distance this would not be possible.

\subsection{Anisotropic Correlation Glyphs}

The rank-2 correlation tensors are always positive semidefinite and symmetric. Thus, they can be decomposed into three orthonormal eigenvectors, which point in the directions of the first, second, and third principal axes of the associated correlation ellipsoid. Regarding the model described in (2), the eigenvalues give the values taken by $\tau$ in the respective eigendirection. The decomposition can be written as

$$
\mathbf{T}=\mathbf{V S V}^{\top},
$$

with matrix V containing the orthonormal eigenvectors (principal components of the ellipsoid) in the columns and the diagonal matrix $\mathbf{S}$ containing the eigenvalues on the diagonal.

As the eigenvectors are not related in general to an isosurface structure in the data, the tensor $\mathbf{T}$ is not directly suitable for analyzing the structural uncertainty of isosurfaces. To overcome this limitation, a basis transformation $\mathbf{T}=\widehat{\mathbf{V}} \widetilde{\mathbf{S}} \widehat{\mathbf{V}}^{\top}$ is performed first. The tensor $\mathbf{T}\left(\mathbf{x}_{i j k}\right)$ at grid point $\mathbf{x}_{i j k}$ is expressed using the normalized data gradient $\mathbf{g}\left(\mathbf{x}_{i j k}\right)$ and two orthonormal vectors $\mathbf{w}_{1}\left(\mathbf{x}_{i j k}\right)$ and $\mathbf{w}_{2}\left(\mathbf{x}_{i j k}\right)$. These two vector are elements of unit vectors in the respective tangent plane

$$
\mathbb{T}\left(\mathbf{x}_{i j k}\right):=\left\{\mathbf{w} \in \mathbb{R}^{3} \mid \mathbf{w}^{\top} \mathbf{g}\left(\mathbf{x}_{i j k}\right)=0,\|\mathbf{w}\|=1\right\} .
$$

The vectors $\mathbf{g}, \mathbf{w}_{1}$, and $\mathbf{w}_{2}$ form the columns of $\widehat{\mathbf{V}}$ and represent the new basis. The new matrix $\widetilde{\mathbf{S}}$ is not diagonal.

With the fixed vector $\mathbf{g}$ there is still one degree of freedom for the orientation of the orthonormal pair $\mathbf{w}_{1}$ and $\mathbf{w}_{2}$ in the tangent plane at point $\mathbf{x}_{i j k}$. We define that $\mathbf{w}_{1}$ and $\mathbf{w}_{2}$ should point in the direction of maximum and minimum $\tau$ in the tangent plane:

$$
\begin{aligned}
& \mathbf{w}_{1}\left(\mathbf{x}_{i j k}\right):=\underset{\mathbf{w} \in \mathbb{T}\left(\mathbf{x}_{i j k}\right)}{\arg \max }\left(\mathbf{w}^{\top} \mathbf{T w}\right) \\
& \mathbf{w}_{2}\left(\mathbf{x}_{i j k}\right):=\underset{\mathbf{w} \in \mathbb{T}\left(\mathbf{x}_{i j k}\right)}{\arg \min }\left(\mathbf{w}^{\top} \mathbf{T w}\right)
\end{aligned}
$$

In order to compute $\mathbf{w}_{1}$ and $\mathbf{w}_{2}$, we first define two arbitrary orthonormal vectors $\mathbf{n}_{1}$ and $\mathbf{n}_{2}$, which span the tangent plane at $\mathbf{x}_{i j k}$, as follows:

$$
\mathbf{n}_{1}:=\frac{\left(g_{2},-g_{1}, 0\right)^{\top}}{\sqrt{g_{1}^{2}+g_{2}^{2}}}, \mathbf{n}_{2}:=\mathbf{g} \times \mathbf{n}_{1} .
$$

Here, $g_{i}$ denotes the $i$ th component of the normalized gradient $\mathrm{g}$. The two spanning vectors are put into the columns of the $3 \times 2$ projection matrix $\mathbf{N}:=\left[\mathbf{n}_{1} \mid \mathbf{n}_{2}\right]$. The tensor $\mathbf{T}$ is projected into the tangent plane by $\mathbf{N}^{\top} \mathbf{T N}$. A singular 
value decomposition of the resulting matrix results in two $2 \mathrm{D}$ orthonormal singular vectors $\mathbf{s}_{1}$ and $\mathbf{s}_{2}$. The vectors $\mathbf{w}_{1}$ and $\mathbf{w}_{2}$ can now be obtained as

$$
\mathbf{w}_{1}=\mathbf{N s}_{1}, \quad \mathbf{w}_{2}=\mathbf{N s}_{2} .
$$

The values of $\tau$ into the three directions are obtained as

$$
\tau_{g}=\mathbf{g}^{\top} \mathbf{T g}, \quad \tau_{1}=\mathbf{w}_{1}^{\top} \mathbf{T} \mathbf{w}_{1}, \quad \tau_{2}=\mathbf{w}_{2}^{\top} \mathbf{T} \mathbf{w}_{2}
$$

Our setting is such that $\mathbf{w}_{1}$ is pointing in the direction of maximum $\tau$. According to (2), this is the direction of minimum correlation. Vector $\mathbf{w}_{2}$ is pointing in the direction of maximum correlation. These two tangent vectors, together with $\mathrm{g}$ and the parameters $\tau_{g}, \tau_{1}$, and $\tau_{2}$, will be used for visualizing the anisotropic correlation relations as well as their absolute strengths.

To visualize anisotropic correlation structures, at every grid point a correlation glyph is constructed from the distinct $3 \mathrm{D}$ orientation given by the isosurface normal $\mathbf{g}$ and the two vectors $\mathbf{w}_{1}$ and $\mathbf{w}_{2}$. This glyph is then used to visualize the correlation ratio in the tangent plane (anisotropy) as well as absolute correlation values for the three directions.

To construct the correlation glyphs, we first have to derive correlation values along the three directions $\mathbf{g}, \mathbf{w}_{1}$, and $\mathbf{w}_{2}$. Therefore, the user selects a specific Euclidean distance $d$ interactively from the interval $\left[0, d_{\text {max }}\right]$, and at every grid point the correlation values are computed as

$$
\rho_{g}=\exp \left(-\tau_{g} d\right), \rho_{1}=\exp \left(-\tau_{1} d\right), \rho_{2}=\exp \left(-\tau_{2} d\right)
$$

So, each glyph visualizes the correlation distribution around its center for the radius $d$. For the construction of the glyph, at every grid vertex a circle is centered at this vertex and aligned with the respective tangent plane. To avoid overlapping glyphs, the circle's radius is set to half the grid spacing.

Within each circle a glyph is placed as shown in Fig. 5(a). Each glyph consists of three zones, where each zone receives a color from the range $[\mathrm{red} \rightarrow$ green], indicating an absolute correlation magnitude value from 0 to 1 . We used the mentioned color map in order to enhance the contrast for absolute correlation values and to relate "stable" regions to "green" and more "unstable" or independent regions to "red."

Zone (1) is a circle with an interactively adjustable radius $r_{1}$. Its color represents the correlation $\rho_{g}$ in the surface normal direction. The colors of zone (2) and (3) encode the values $\rho_{2}$ for maximum and $\rho_{1}$ for minimum correlation in the tangent plane. Zone (3) always forms a circle with a fixed radius $r_{3}$. Zone (2) is an ellipse, which is oriented along the maximum correlation axis $\mathbf{w}_{2}$ and has an radius in this direction always equal to $r_{3}$. The radius in direction $\mathbf{w}_{1}$ is equal to $\left[1-\left(\rho_{1} / \rho_{2}\right)\right] r_{1}+\left(\rho_{1} / \rho_{2}\right) r_{3}$. So, the alignment of the ellipse with respect to the radii $r_{1}$ and $r_{3}$ directly visualizes the correlation ratio in the tangent plane. Compare the ellipse variations in Fig. $5(\mathrm{~b})$. Note that if $\rho_{1}=\rho_{2}$, then zone (2) fully covers zone (3).

The introduced correlation glyphs have several strong advantages, oriented on the requirements presented in Section 4.1: First, it is possible to show the correlation anisotropy in the tangent plane as well as absolute correlation

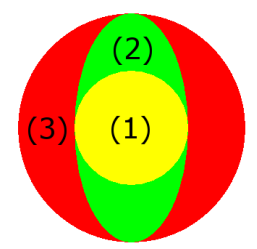

(a)

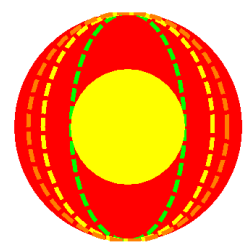

(b)

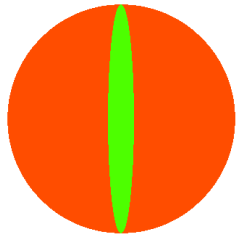

(c)

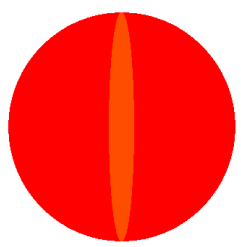

(d)

FIG. 5: In (a) the used correlation glyph is shown. Absolute correlation strengths are mapped from $[0,1]$ to [red $\rightarrow$ green] for surface normal (1), first (2) and second (3) principal tangential correlation directions in three zones. (b) Geometry of zone (2) visualizes the correlation ratio between the two tangential direction. (c) Glyph for high contrast tangential correlation values 0.9 (2) and 0.1 (3) is shown. (d) Glyph for low contrast tangential correlation values 0.09 (2) and 0.01 (3) is shown. 
values (coded in color in the three zones) simultaneously in one picture. Showing only correlation ratios would not be sufficient. For instance, the two correlation values 0.9 and 0.1 have a ratio of $9: 1$, but 0.09 and 0.01 would also have a ratio of 9:1! As the user is predominantly interested in ratios where the absolute difference between both values is also large ( 0.8 compared to 0.08 in our example), the second case is not really of interest.

The two cases are shown with our glyph visualization in Figs. 5(c) and 5(d). In both cases, the geometry and the radii of the elliptic zone (2) are the same, but due to the lower color contrast in Fig. 5(d) (colors indicate correlations 0.01 and 0.09 ), the ellipse is almost vanishing. So, the anisotropy is only visible in regions where $\left|\rho_{1}-\rho_{2}\right|$ is considerably large and the user's focus is not distracted by glyphs indicating high ratios of very low correlation values. This is a strong advantage in comparison to other tensor-glyph visualization approaches!

Furthermore, the user is able to adjust the radius $r_{1}$ of zone (1) between 0 and $r_{3}$. For $r_{1}=0$ the elliptic zone (2) has the largest range of variations. This is best for illustrating the correlation anisotropy in the tangent plane. For $r_{1}=r_{3}$ zone (1) covers zone (3) completely, zone (2) is not perceivable anymore, and only the absolute correlation value in the surface normal direction is visualized. So, while interactively changing the radius $r_{1}$, the user has the possibility of putting the focus either on the analysis of the correlation anisotropy along the surface or the strengths in normal direction —or a mixture of both!

If the structural variability of an isosurface in a 3D scalar field for a particular isovalue $\theta$ has to be analyzed, not for every grid vertex should a correlation glyph be visualized. Instead, the visualization should be restricted to only those glyphs close to the surface. Therefore, we make the visualization of a glyph dependent on the mean value $\mu\left(\mathbf{x}_{i j k}\right)$ at the respective grid vertex: the glyph at position $\mathbf{x}_{i j k}$ is rendered if

$$
\begin{aligned}
& \theta \geq \min \left(\left\{\mu\left(\mathbf{x}_{i j k}\right), \mu\left(\mathbf{x}_{i \pm 1 j k}\right), \mu\left(\mathbf{x}_{i j \pm 1 k}\right), \mu\left(\mathbf{x}_{i j k \pm 1}\right)\right\}\right) \\
& \theta \leq \max \left(\left\{\mu\left(\mathbf{x}_{i j k}\right), \mu\left(\mathbf{x}_{i \pm 1 j k}\right), \mu\left(\mathbf{x}_{i j \pm 1 k}\right), \mu\left(\mathbf{x}_{i j k \pm 1}\right)\right\}\right) .
\end{aligned}
$$

This rule was chosen to reduce overlapping of glyphs. The regular placing of the glyphs at the vertices of the 3D grid facilitates the visual perception of anisotropic correlation contrasts compared to approaches where the glyphs have variable distances between each other.

\subsection{Discussion}

To validate the effectiveness of our proposed visualization technique for analyzing the structural variability of isosurfaces in uncertain 3D scalar fields, we have conducted two experiments using different data sets: A stochastically simulated data set with a synthetic setting (see Fig. 3) and a real-world data set comprised of an ensemble of simulated weather forecasts.

In Fig. 3, we have demonstrated the structural variability of an isosurface depending on different correlation settings. Figure 6 shows the visualization of the assigned correlation structures using our glyph-based visualization approach. Here, the glyph geometry clearly reveals the correlation ratios. In region (1), the glyphs indicate an isotropic correlation distribution. In (2) and (3), the directions along which the correlation is high can be clearly perceived. In region (4), the occurrence of glyphs having green center zones surrounded by red zones indicates a high ratio between the correlations in $x / y$ direction (tangent plane, low correlation) and the surface normal direction (high correlation). The reverse behavior is visualized in region (5), i.e., low correlation along the normal direction.

The visualization in Fig. 7 shows a 3D temperature field in the exosphere above Europe and the North Atlantic Ocean in spherical coordinates. It contains the mean values of multiple fields that were simulated by the European Center for Medium-Range Weather Forecasts using slightly different input parameters. The image Fig. 7(a) shows an isosurface in the mean temperature field. In Fig. 7(b), our glyph-based correlation visualization is used to depict structural variation effects. In Fig. 7(c), a close-up view on the region marked by the white rectangle is shown. The green glyph center and the red outer zones indicate a high ratio between the correlation strengths in the tangential and normal directions. In such regions, a higher stochastic independence between points in the tangential plane can be concluded. The surrounding regions, which are covered by homogeneous green glyphs, are much less likely affected by structural uncertainty.

Figure 7(d) and 7(e) reveal the interplay between correlation ratios and color-based coding of correlation strengths. In (d), only the correlation anisotropy in the tangent planes is visualized, but the correlation in the normal direction is 


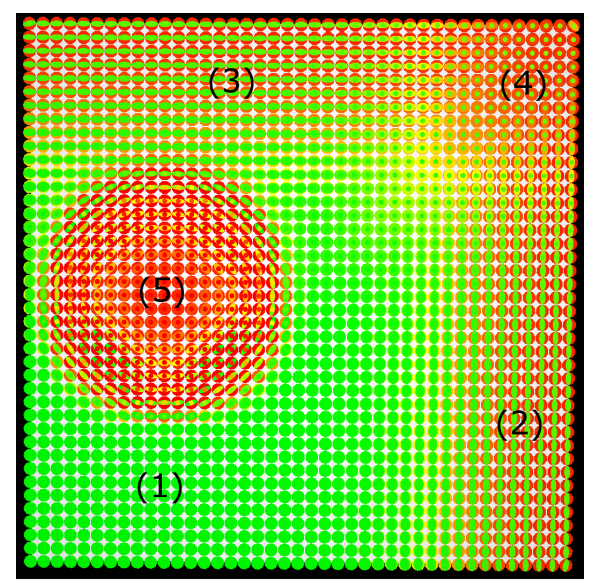

FIG. 6: Top view on correlation visualization on mean surface from example 3 (Fig. 3). Strong local correlations in (1) indicate low structural uncertainty. Glyph geometries reveal high anisotropic correlation difference for regions (2) and (3). High contrast between strong correlation strengths in normal direction and lower ones in tangential directions are shown in (4). Region (5) is affected by low normal correlation, indicated by red glyph center zones.
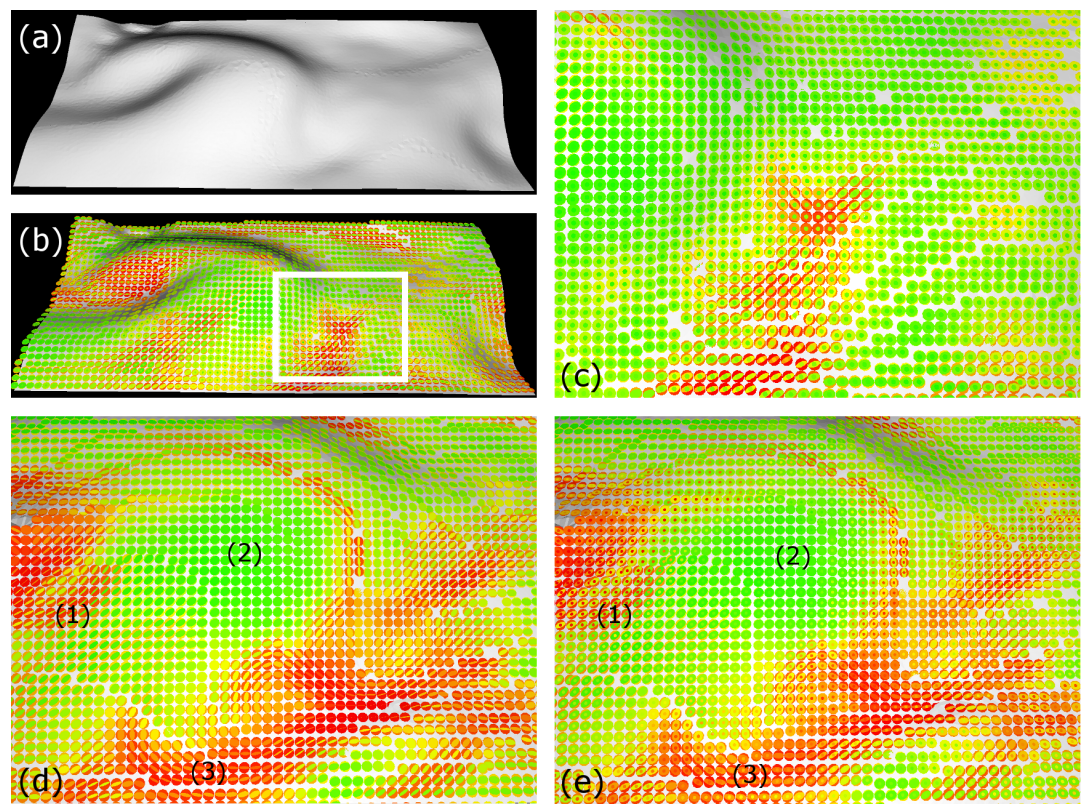

FIG. 7: In (a) a mean temperature isosurface in a 3D scalar ensemble weather forecast data set is shown. (b) Correlation glyphs reveal anisotropic correlation structures in the surface's vicinity. (c) Close-up view of rectangular region shows region with low tangential correlation (red outer glyph zones) and strong normal correlation values (green inner zone). In (d), an region with strong homogeneous correlation and low structural uncertainty thereof (2) is visually separated from low and anisotropic correlation structures in (1) and (3). (e) Additional correlation values in the surface normal direction are coded in color in glyph center zones.

ignored. For a tongue-shaped region around (2), strong homogeneous local correlation close to 1 and low structural uncertainty is indicated by uniformly colored green glyphs. In region (1), high contrast between the two glyph zones (green and red) indicates a strong correlation anisotropy [almost 1 for the first tangential direction (green) and almost 0 for the second tangential direction (red)]. Although the glyph geometry reveals the same correlation ratio as, for 
instance, in region (3), the color contrast between the two glyph zones emphasizes the absolute correlation differences. In Fig. 7(e), we also show the interior glyph zones for the absolute correlation values in the normal direction. One can observe that there is very low correlation along the normal direction around the stable homogeneous region (2), indicated by the red center zones. This low correlation in the normal direction indicates rather high stochastic instability, meaning that isosurfaces in these regions are very likely to separate into multiple disconnected parts [cf. region (5) in Fig. 3(c)].

From the given examples some general advantages of the proposed glyph-based approach (based on the anisotropic correlation model) for correlation visualization can be concluded:

1. The local distance-dependent correlation model, used to parametrize the glyph shape and color, allows for a memory reduction to $O(n)$ correlation/tensor values for $n$ grid points.

2. The correlation parameter tensor model accounts for the anisotropic nature of local correlation strengths.

3. The glyph geometry clearly indicates correlation ratios in the surface tangent planes and effectively emphasizes the predominant correlation direction.

4. The color-mapping scheme intuitively reveals the absolute correlation values in the surface normal and the two tangential directions. It further allows for differentiating between interesting anisotropic correlation regions (low and high correlation in tangent plane) and less interesting regions where the correlation in both directions is low, which however could have the same ratios [cf. Fig. 5(c) and 5(d)].

5. Since the glyph design (variable radius for normal correlation zone) can be changed interactively, the user can flexibly change the focus between a visualization, more concentrated on the anisotropic structures in the tangent planes or on anisotropic structures between the tangent plane and the surface normal direction. So, the structural uncertainty can be assessed interactively for different spatial directions.

Despite the advantages, however, our approach also suffers from some limitations and leaves several open questions and requirements:

1. Our approach does not show any absolute uncertainty information like standard deviation, which is mandatory for a more comprehensive uncertainty analysis. Here, a thorough analysis of an adequate visualization channel (color, opacity, etc.) would be necessary, as a straightforward merge with an intuitive uncertainty visualization approach, e.g., shown in [25], is often not possible.

2. The glyph-based approach is based on a local correlation model and thus can only show the correlation to regions close to the position where a glyph is placed. Global correlation structures are ignored.

3. Inverse correlation structures cannot be visualized, as the chosen correlation model only accounts for correlations strengths (magnitudes). Here a global correlation model and a different color scheme would be necessary.

4. Both the glyph geometry and the color coding refer to the correlation between the values in a certain distance to each other. Even though this distance can be selected interactively and arbitrarily, it does not appear in the visualization.

From the analysis of the advantages and limitations of the glyph-based approach for correlation visualization, it can be concluded that the design of techniques allowing for an intuitive and effective correlation visualization is not straightforward. The integration of correlation information into visualizations of complicated multidimensional structures is extremely challenging due to the limited number of visualization channels (color, opacity, geometry, etc.). Although our approach cannot fulfill all requirements on a correlation visualization technique, it can be seen as a first step toward such an integration and is meant to stimulate further research in this direction. 


\section{CONCLUSION}

In this paper, we provided basic insights into the concept of correlations in uncertain scalar fields. Furthermore, we stressed the importance for integrating correlation data into uncertainty visualization approaches in order to make more reliable assumptions on the structural uncertainty of features and to get a more comprehensive understanding of the effects uncertainty exerts on the underlying data set. In addition, we gave an overview on important features, which should be integrated into a correlation visualization concept.

In order to approach some of the proposed requirements, we presented a glyph-based correlation visualization approach. Therefore, we suggested a tensor-based distance-dependent correlation model for modeling local anisotropic correlation strengths. This model was used for generating glyphs, specially adapted to isosurface structures and indicating correlation ratios among tangential and between tangential and normal surface directions. A color-mapping scheme was introduced, allowing an interactive perception of absolute correlation strengths in various spatial directions. In a synthetic and one real-world data set, we showed the effectiveness of our approach for identifying regions with high and low structural uncertainty.

In the future, we will pursue further research on visual approaches for illustrating the interplay between correlation structures and uncertainty magnitudes. The proposed correlation model could be used for computing parameter tensors for different sampling point neighborhoods and distances. This would allow a level-of-detail correlation visualization approach resulting in a both local as well as global correlation analysis. Finally, a more thorough analysis of further approaches for inverse correlation visualization would be needed for a more complete global correlation understanding.

\section{REFERENCES}

1. Feller, W., An Introduction to Probability Theory and Its Applications, Vol. 2, Wiley, India, 2008.

2. Georgii, H., Stochastics: Introduction to Probability and Statistics, Walter De Gruyter, Berlin, 2008.

3. Rodgers, J. and Nicewander, W., Thirteen ways to look at the correlation coefficient, Am. Stat., 42(1):59-66, 1988.

4. Johnson, C. and Sanderson, A., A next step: Visualizing errors and uncertainty, IEEE Trans. Comput. Graphics Appl., 23(5):610, 2003.

5. Pang, A., Wittenbrink, C., and Lodha, S., Approaches to uncertainty visualization, Visual Comput., 13(8):370-390, 1997.

6. European Centre for Medium-Range Weather Forecasts, Demeter temperature ensemble, Available online at http://www.ecmwf.int.

7. Fornasini, P., The Uncertainty in Physical Measurements: An Introduction to Data Analysis in the Physics Laboratory, Springer Verlag, Berlin, 2008.

8. Tarantola, A., Inverse Problem Theory and Methods for Model Parameter Estimation, Society for Industrial Mathematics, Philadelphia, 2005.

9. Ayyub, B. and Klir, G., Uncertainty Modeling and Analysis in Engineering and the Sciences, Boca Raton, FL, CRC Press, 2006.

10. MacEachren, A., Robinson, A., Hopper, S., Gardner, S., Murray, R., Gahegan, M., and Hetzler, E., Visualizing geospatial information uncertainty: What we know and what we need to know, Cartog. Geogr. Inf. Sci., 32(3):139-161, 2005.

11. Bostrom, A., Anselin, L., and Farris, J., Visualizing seismic risk and uncertainty, Ann. N. Y. Acad. Sci., 1128(1):29-40, 2008.

12. Li, H., Fu, C., Li, Y., and Hanson, A., Visualizing large-scale uncertainty in astrophysical data, IEEE Trans. Visualization Comput. Graphics, 13(6):1640-1647, 2007.

13. Thomson, J., Hetzler, E., MacEachren, A., Gahegan, M., and Pavel, M., A typology for visualizing uncertainty, In Proceedings SPIE, Vol. 5669, pp. 146-157, Citeseer, 2005.

14. Griethe, H. and Schumann, H., The visualization of uncertain data: Methods and problems, In Proceedings of SimVis, pp. 143-156, SCS, Magdeburg, Germany, 2006.

15. Potter, K., Access date: 07/24/2012, http://www.sci.utah.edu/ kpotter/library/uncertainVis/index.html.

Volume 3, Number 2, 2013 
16. Wittenbrink, C., Pang, A., and Lodha, S., Glyphs for visualizing uncertainty in vector fields, IEEE Trans. Visualization Comput. Graphics, 2(3):266-279, 2002.

17. Djurcilov, S., Kim, K., Lermusiaux, P., and Pang, A., Visualizing scalar volumetric data with uncertainty, Comput. Graphics, 26(2):239-248, 2002.

18. Rhodes, P., Laramee, R., Bergeron, R., and Sparr, T., Uncertainty visualization methods in isosurface rendering, In Eurographics, pp. 83-88, Citeseer, 2003.

19. Lundstrom, C., Ljung, P., Persson, A., and Ynnerman, A., Uncertainty visualization in medical volume rendering using probabilistic animation, IEEE Trans. Visualization Comput. Graphics, 13(6):1648-1655, 2007.

20. Sanyal, J., Zhang, S., Dyer, J., Mercer, A., Amburn, P., and Moorhead, R., Noodles: A tool for visualization of numerical weather model ensemble uncertainty, IEEE Trans. Visualization Comput. Graphics, 16(6):1421-1430, 2010.

21. Zehner, B., Watanabe, N., and Kolditz, O., Visualization of gridded scalar data with uncertainty in geosciences, Comput. Geoscie., 36(10):1268-1275, 2010.

22. Kindlmann, G., Whitaker, R., Tasdizen, T., and Moller, T., Curvature-based transfer functions for direct volume rendering: Methods and applications, In Visualization, pp. 513-520, IEEE, 2003.

23. Grigoryan, G. and Rheingans, P., Point-based probabilistic surfaces to show surface uncertainty, IEEE Trans. Visualization Comput. Graphics, 10(5):564-573, 2004.

24. Brown, R., Animated visual vibrations as an uncertainty visualisation technique, In Graphite, pp. 84-89, ACM, 2004.

25. Pfaffelmoser, T., Reitinger, M., and Westermann, R., Visualizing the positional and geometrical variability of isosurfaces in uncertain scalar fields, In Computer Graphics Forum, Vol. 30, pp. 951-960, Wiley Online Library, 2011.

26. Pöthkow, K., Weber, B., and Hege, H., Probabilistic marching cubes, In Computer Graphics Forum, Vol. 30, pp. 931-940. Wiley Online Library, 2011.

27. Pöthkow, K. and Hege, H., Positional uncertainty of isocontours: Condition analysis and probabilistic measures, IEEE Trans. Visualization Comput. Graphics, (99):1-1, 2010.

28. Jen, D., Parente, P., Robbins, J., Weigle, C., Taylor II, R., Burette, A., and Weinberg, R., Imagesurfer: A tool for visualizing correlations between two volume scalar fields, In Proceedings of the IEEE Conference on Visualization, pp. 529-536, IEEE, Austin, TX, 2004.

29. Kindlmann, G., Weinstein, D., Lee, A., Toga, A., and Thompson, P., Visualization of anatomic covariance tensor fields, In Engineering in Medicine and Biology Society, IEMBS'04. 26th Annual International Conference of the IEEE, Vol. 1, pp. 1842-1845, IEEE, San Francisco, CA, 2004.

30. Yang, C., Xiu, D., and Kirby, R. M., Visualization of covariance and cross-covariance fields, Int. J. Uncertainty Quantific., 3(1):25-38, 2013.

31. Pfaffelmoser, T. and Westermann, R., Visualization of global correlation structures in uncertain 2D scalar fields, In Computer Graphics Forum, Vol. 31, Wiley Online Library, 2012.

32. Nolet, G., A Breviary of Seismic Tomography: Imaging the Interior of the Earth and Sun, Cambridge, Cambridge University Press, 2008.

33. Björck, Å., Numerical Methods for Least Squares Problems, Society for Industrial Mathematics, Philadelphia, 1996. 